\title{
Multi-task Ranking SVM for Image Cosegmentaiton
}

\author{
Xianpeng Lianga ${ }^{\mathrm{a}}$ Lin Zhu ${ }^{\mathrm{a}}$, De-Shuang Huang, ${ }^{\mathrm{a}, *}$ \\ a Institute of Machine Learning and Systems Biology, School of Electronics and Information Engineering, Tongji University, \\ Caoan Road 4800, Shanghai 201804, China.
}

\begin{abstract}
Cosegmentation is the task of simultaneously segmenting multiple images that contain common or similar foreground objects. The assumption that common objects appear in multiple images provides a weak form of supervised prior information, thus cosegmentation usually performs better than unsupervised segmentation methods. Recently, a multi-task learning based cosegmentation method was proposed, and it can simultaneously segment more than two images and easily add different types of prior. However, it has the shortcoming of information loss in the initialization of the multi-task classification model. To ameliorate this problem, in this paper, we propose a novel multi-task ranking SVM model which incorporates multi-task learning and learning to rank into a unified framework. The proposed model is trained using the relative order information between the cosaliency score of pixel pairs. In addition, an optimization algorithm is proposed to optimize the multi-task ranking SVM model based on the alternative direction method of multipliers (ADMM), which ensures that the proposed method is faster than most of state-of-the-art cosegmentation approaches. Finally, the proposed method is evaluated on two widely used benchmark datasets, i.e. CMU iCoseg and MSRC. The experiment results show that the proposed approach is effective and performs better than most of the state-of-the-art works.
\end{abstract}

Keywords: Image cosegmentation, Multi-task learning, Ranking SVM, Visual saliency, ADMM

\section{Introduction}

Image cosegmentation is the task of simultaneously segmenting multiple related images into different parts. Different with unsupervised single image segmentation approaches, image cosegmentation makes the assumption that these related images contain common or similar objects, which provides a form of weakly supervised prior information. Therefore, image cosegmentation can usually achieve better performance than those unsupervised single image segmentation approaches.

The idea of cosegmentation was first introduced in [1], in which a MRF based generative model was proposed to simultaneously segment two images. The work [2] later proposed a discriminative cosegmentation model which can simultaneously segment more than two images and deal with more greater variation foreground appearance. The work [3] tried to deal with a more challenge cosegmentation task, multiple objects cosegmentation. The works $[4,5]$ used the supervised image annotation to do cosegmentation. The above methods can be considered as low level feature based methods[6, 7]. Recently, some object-based cosegmentation methods are proposed, and the details of these works can be found in [8-11].

There are several limits in the above works. The work [1] can segment only two images. Although [2] can segment more than two images, its computational complexity is too high. The methods [4, 5] need fully supervised human annotation. In order to solve these limitations, the work [12] proposed a multi-task learning

\footnotetext{
${ }^{*}$ Corresponding author. E-mail address: dshuang@tongji.edu.cn
} 
based image cosegmentation method. In this framework, the segmentation of each image is treated as a single task with independent classifier. The prior information shared among these images is modeled by the multi-task regularization term. Specifically, features are firstly extracted for each image, and then these features are used to compute the cosaliency score of these images, in which the computed cosaliency score is in the interval $[0,1]$. Third, the cosaliency score is binarized by a threshold, and then these binarized score is used to initialize the multi-task classification model. Finally, the output of multi-task classification model is used to initialize the GrabCut algorithm which outputs the final segmentation results.

The above multi-task learning based cosegmentation model has some merits. First, it can simultaneously segment more than two images. Second, its computational complexity is lower than previous methods when segmenting more than two images. Third, it's easy and flexible to model the shared prior information among these images by the multi-task regularization term. However, there is still weak points in [12]. In particular, after the cosaliency score of those images is computed, it is binarized by a fixed threshold and used to initialize the multi-task classifier model. However, it is difficult to choose the fixed threshold. Inappropriate threshold may lead to information loss. Thus how to combine the cosaliency score with the framework of multi-task learning remains a problem.

In this paper, we focus on the framework of multi-task learning based cosegmentation methods and propose a novel multi-task Ranking SVM (MTR-SVM) model to solve the above problems. The proposed MTR-SVM is based on the frameworks of both multi-task learning [13-15] and learning to rank [16-18], and is directly trained using the relative order information between the cosaliency score of pixel pairs instead of the binarzed form of cosaliency score. MTR-SVM can flexibly model the relationships or shared features among these tasks by the regularization term. In addition, we propose an ADMM based algorithm to optimize the MTR-SVM model and it ensures that MTR-SVM is faster than those state-of-the-art cosegmentation approaches. Finally, we test the performance of the proposed approach on two widely used benchmark datasets, the CMU iCoseg dataset and the MSRC dataset. The experiment results show that the proposed approach improves the segmentation accuracy significantly and costs less computational time, as compared with recent state-of-the-art cosegmentation techniques.

The rest of this paper is organized as follows. Section 2 introduces the related works and the MTR-SVM model is proposed on Section 3. Section 4 designs a novel ADMM based learning algorithm for MTR-SVM model. The experiment evaluation is given in Section 5. The conclusion and further works are discussed in Section 6.

\section{Related works}

\subsection{Learning to rank}

Learning to rank $[17,18]$ is a popular method in Information Retrieval and it aims to learn a ranking function from the training data. The existing learning to rank algorithms can be roughly divided into three categories: the point-wise, pair-wise and list-wise approaches. The work [19] proposed a regression based point-wise ranking model which learns a regression function between the ranking score and the features. A SVM based pair-wise ranking model was introduced in [16], where the ranking problem is transformed into a two class classification problem. In [20], the list of objects is treated as an instance, and a list-wise loss function on the basis of probability model is formulated. Besides, there are other works about ranking models, and these can be found in $[21,22]$.

\subsection{Multi-task learning}

Multi-task learning $[14,15]$ is the task of simultaneously learning multiple related tasks by exploiting task 
relationships or shared features among these tasks. Multi-task learning usually can achieve better performance than single-task learning methods, especially when the number of training data of each task is limited. The current multi-task learning methods can be divided into two categories: methods are based on sharing task relationships [23] and that are based on sharing features [13]. Most of multi-task learning approaches share the common information by the multi-task regularization term. The work [14] proposed a low rank multi-task learning method which assumes that the parameter vectors of those tasks are located in a low rank subspace. In [13], a $\ell_{2,1}$ regularization term is used to constrain the parameter matrix, so that the parameter vectors of each task share common features. The work [23] proposed a kernel function based multi-task learning model which can automatically learn the task relationships.

\section{Multi-Task ranking SVM for image cosegmentation}

In this part, we introduce a novel multi-task Ranking SVM model for image cosegmentation. The general framework of the proposed method is illustrated in figure 1. In order to improve the computational speed, SLIC [24] is used to over-segment each image into 200 superpixels.

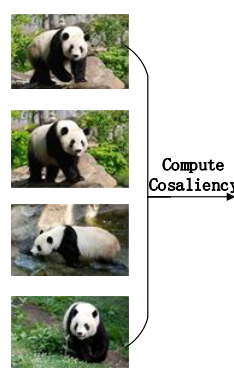

(a)

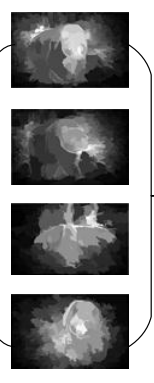

(b)

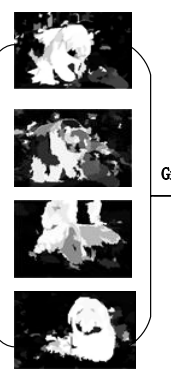

(c)

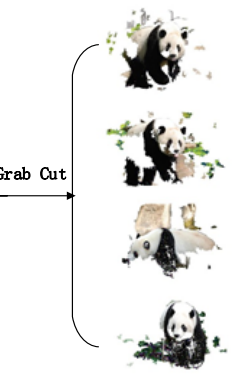

(d)

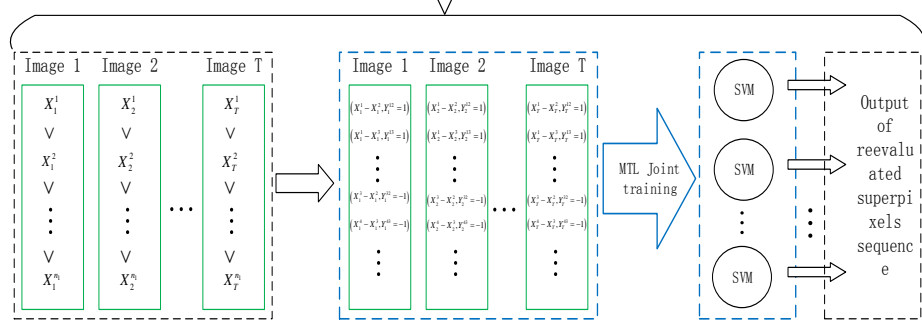

Pairwise training instance

Fig. 1 The overview of the proposed algorithm. The up row shows the segmentation flow and the down row shows the specific structure of the proposed MTR-SVM model.

\subsection{Feature extraction and visual cosaliency}

In this part, color features, texture features, geometry measurements and edges features are firstly extracted for each superpixel [25]. And we use the symbol $X_{i}^{j}$ to represent the extracted features of the $j$-th superpixel in $i$-th image. Then the similarities between superpixels within each image and across different images are computed, and it is useful to the computation of the cosaliency. We use the symbol $A(i, j ; p, q)$ to represent the similarity between the $j$-th superpixel in $i$-th image and $q$-th superpixel in $p$-th image.

Visual saliency is the task of finding the visual attention parts of the whole visual field and has attracted a lot of researches [26-29]. Visual saliency is usually used to initialize the foreground parts in image segmentation task. 
For multiple images, visual cosaliency aims to find the saliency part that is not only salient in its own image but also similar to salient part in other images. In this paper, the cosaliency for each image is computed by the robust visual cosaliency method proposed in [12]. More specifically, symbol $s_{i}^{j}$ is used to represent the cosaliency score of superpixel $X_{i}^{j}$ and is computed by an iteration method,

$$
s_{i}^{j}(t+1)=(1-\alpha) \hat{s}_{i}^{j}+\alpha \sum_{p, q: s_{p}^{q}(t) \geq \tau} s_{p}^{q}(t) \times A(i, j ; p, q)
$$

where $s_{i}^{j}(t+1)$ is the cosaliency score of $X_{i}^{j}$ in the $t+1$ iteration. $\hat{s}_{i}^{j}$ is the saliency score of $X_{i}^{j}$ and is computed by a PCA based saliency computation method [26]. $\tau$ is a threshold. $\alpha$ is a weight parameter and is in interval $[0,1]$.

\subsection{MTR-SVM model}

When the cosaliency score of each image has been computed, we choose a pair-wise learning to rank model (ranking SVM) to directly fit these cosaliency score, instead of binarizing the cosaliency score to initialize the multi-task classification model in [12]. And the learning to rank based method can avoid the difficult choice of the threshold and reduce information loss.

Specifically, for a single image $i$, assume that there are $n_{i}$ superpixels. Then a linear ranking function $f_{i}(x)=\left\langle W_{i}, x\right\rangle$ will be learned to fit these features and cosaliency score of superpixels in image $i$, in which $W_{i}$ is the parameter vector holding the same dimension with feature vector $x$. In addition, function $f_{i}$ should keep the ranking order of these superpixels, which can be formulated as the following constraints:

$$
s_{i}^{j}>s_{i}^{k} \Leftrightarrow f_{i}\left(X_{i}^{j}\right)>f_{i}\left(X_{i}^{k}\right) \Leftrightarrow\left\langle W_{i}, X_{i}^{j}-X_{i}^{k}\right\rangle>0
$$

The constraints in Eq. (2) are equivalent to a two-class classification problem with the following training instances:

$$
\begin{aligned}
& \left\{\left(X_{i}^{j}-X_{i}^{k}, Y_{i}^{j k}\right), j, k \in\left\{1,2, \cdots, n_{i}\right\}\right\} \\
& \text { where } Y_{i}^{j k}= \begin{cases}+1, & \text { if } s_{i}^{j}>s_{i}^{k} \\
-1, & \text { otherwise }\end{cases}
\end{aligned}
$$

Next, we utilize the SVM model to fit these classification data. And the objective function of the classification problem can be written as:

$$
\begin{aligned}
\min _{w_{i}} & \frac{1}{2}\left\|W_{i}\right\|^{2}+C \sum_{j, k=1}^{n_{i}} \xi_{j, k} \\
\text { s.t. } & \xi_{j, k} \geq 0 \\
& Y_{i}^{j, k}\left\langle W_{i}, X_{i}^{j}-X_{i}^{k}\right\rangle \geq 1-\xi_{j, k}, j, k \in\left\{1, \cdots, n_{i}\right\}
\end{aligned}
$$

Note that Eq. (4) is equivalent to Eq. (5):

$$
\min _{w_{i}} \sum_{j, k=1}^{n_{i}}\left[1-Y_{i}^{j, k}\left\langle W_{i}, X_{i}^{j}-X_{i}^{k}\right\rangle\right]_{+}+\lambda\left\|W_{i}\right\|^{2}
$$

where $\lambda=\frac{1}{2 C}$, subscript " + " means the positive part. 
After Eq. (5) is optimized, we use the symbol $W_{i}^{*}$ to represent the optimized parameter vector. Then the ranking score of superpixel $X_{i}^{j}$ can be reevaluated by $f_{i}\left(X_{i}^{j}\right)=\left\langle W_{i}^{*}, X_{i}^{j}\right\rangle$.

The segmentation of each image can be considered as a single task, and the segmentation of multiple images can be treated as a multi-task segmentation problem. The prior information that these images share same or similar foreground objects can be modeled by the regularization term of the multi-task learning models. Assuming there are $T$ similar images needed to be segmented, and for each image $t$, there are $n_{t}^{2}-n_{t}$ different pairwise instances $\left\{\left(X_{t}^{j}-X_{t}^{k}, Y_{t}^{j k}\right), j, k \in\left\{1,2, \cdots, n_{t}\right\}\right\}$. Then the general formula of regularization multi-task learning is:

$$
\min _{W} \sum_{t=1}^{T} \sum_{j, k=1}^{n_{t}} L\left(Y_{t}^{j k}, f_{t}\left(X_{t}^{j}-X_{t}^{k}\right)\right)+\lambda_{1}\|W\|_{2}^{2}+\lambda_{2} R(W)
$$

where $W_{i}$ is the parameter vector of $i$-th task and it has the same dimension with feature vector $X_{i}^{j}$ $W=\left[W_{1}, W_{2}, \cdots, W_{T}\right]$ is the parameter matrix. $L(\cdot, \cdot)$ is the loss function. Here we use hinge loss function. $R(\cdot)$ is the multi-task regularization term. In this paper, three types of regularization terms are used, Mean graph regularization, $R(W)=\sum_{t=1}^{T}\left\|W_{t}-\frac{1}{T} \sum_{s=1}^{T} W_{s}\right\|_{2}^{2}$, which assumes that the task parameter vectors are drawn from the same distribution and all of them are close to their mean; Low rank regularization, $R(W)=\|W\|_{*}$, which assumes that the task parameter vectors share a common low rank subspace, and each parameter vector can be linearly represented by others; $\ell_{2,1}$ regularization, $R(W)=\|W\|_{2,1}$, which assumes that the task parameter vectors share common features.

Finally, combining Eq. (5) with Eq. (6), the object function of Multi-task Ranking SVM model is formulated as follows:

$$
\min _{W} \sum_{t=1}^{T} \sum_{j, k=1}^{n_{t}}\left[1-Y_{t}^{j k}\left\langle W_{t}, \mathrm{X}_{t}^{j}-\mathrm{X}_{t}^{k}\right\rangle\right]_{+}+\lambda_{1} \sum_{t=1}^{T}\left\|W_{t}\right\|^{2}+\lambda_{2} R(W)
$$

For the $t$-th image, the pairwise training dataset $\left\{\left(X_{t}^{j}-X_{t}^{k}, \mathrm{Y}_{t}^{j k}\right), j, k \in\left\{1,2, \cdots, n_{t}\right\}\right\}$ totally contains $n_{t}^{2}-n_{t}$ different training instances. And in the following, for the convenience of deduction, we use $\left\{\left(x_{t}^{i}, y_{t}^{i}\right), i \in\left\{1,2, \cdots, \mathrm{N}_{t}\right\}\right\} \quad$ to represent the training dataset $\left\{\left(X_{t}^{j}-X_{t}^{k}, \mathrm{Y}_{t}^{j k}\right), j, k \in\left\{1,2, \cdots, n_{t}\right\}\right\}$, where $N_{t}=n_{t}^{2}-n_{t}$. Thus, Eq. (7) can be rewritten as,

$$
\min _{W} \sum_{t=1}^{T} \sum_{i=1}^{N_{t}}\left[1-y_{t}^{i}\left\langle W_{t}, \mathrm{x}_{t}^{i}\right\rangle\right]_{+}+\lambda_{1} \sum_{t=1}^{T}\left\|W_{t}\right\|^{2}+\lambda_{2} R(W)
$$

In the following section, we propose an ADMM based optimization method to optimize the above object function. After the MTR-SVM model has been optimized, the ranking function of each image can be achieved. Then the saliency score of superpixels in all images can be reevaluated by applying the ranking function to these 
superpixels, $f_{t}\left(X_{t}^{i}\right)=\left\langle W_{t}, X_{t}^{i}\right\rangle$. Lastly, the GrabCut algorithm is used to achieve the final segmentation results [30], and it can be initialized by the following method: for each superpixel $X_{t}^{i}$, if its score is smaller than $80 \%$ of the mean scores of all the superpixels in all images, it will be labeled as background.

\section{The alternative direction method of multipliers for MTR-SVM model}

In this section, the alternative direction method of multipliers (ADMM) [31-35] is used to optimize the problem in Eq. (8). Although the alternative direction method has been developed for decades of years, how to use it to solve a specific problem is not mathematically trivial. In the following, we derive the solution of the problem in Eq. (8).

\subsection{ADMM}

Consider the following optimization problem:

$$
\begin{aligned}
& \min _{x, y} f_{1}(x)+f_{2}(y) \\
& \text { s.t. } A x+B y=c
\end{aligned}
$$

where $x \in R^{n_{1}}$ and $y \in R^{n_{2}}$ are optimization variables, $A \in R^{m \times n_{1}}$ and $B \in R^{m \times n_{2}}$ are linear operators, $c \in R^{m}$ is a vector of data. ADMM is an iterative method to solve problem in Eq. (9) and it minimizes the following augmented Lagrangian:

$$
\begin{aligned}
L(x, y, \lambda)= & f_{1}(x)+f_{2}(y)-\langle\lambda, A x+B y-c\rangle \\
& +\frac{\tau}{2}\|c-A x-B y\|^{2}
\end{aligned}
$$

where $\tau$ is a penalty parameter and $\lambda$ is the Lagrange multiplier. Then Eq. (10) is equivalent to the following two sub-problems:

$$
\begin{aligned}
& \min _{x} f_{1}(x)-\langle\lambda, A x+B y-c\rangle+\frac{\tau}{2}\|c-A x-B y\|^{2} \\
& \min _{y} f_{2}(y)-\langle\lambda, A x+B y-c\rangle+\frac{\tau}{2}\|c-A x-B y\|^{2}
\end{aligned}
$$

The updating rule of the Lagrange multiplier is: $\lambda_{\text {new }}=\lambda_{\text {old }}-\tau(A x+B y-c)$. The complete steps of ADMM are described in algorithm 1.

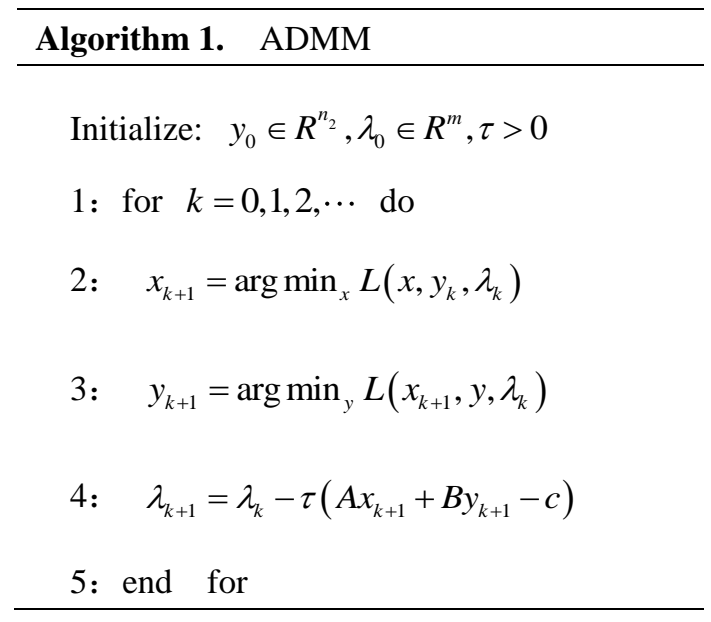


4.2 ADMM to solve problem in Eq. (8) with mean graph regularization

In this section, we derive the optimization problem in Eq. (8) with the mean graph multi-task regularization: $R(W)=\sum_{t=1}^{T}\left\|W_{t}-\frac{1}{T} \sum_{s=1}^{T} W_{s}\right\|_{2}^{2}$. First, we use an auxiliary variable $e_{t}^{j}=y_{t}^{j}-\left\langle W_{t}, x_{t}^{j}\right\rangle, t=1, \cdots, T ; j=1, \cdots, \mathrm{N}_{t}$ to transform Eq. (8) into the following form:

$$
\begin{aligned}
& \min _{W} \sum_{t=1}^{T} \sum_{j=1}^{\mathrm{N}_{t}}\left[y_{t}^{j} e_{t}^{j}\right]_{+}+\lambda_{1} \sum_{t=1}^{T}\left\|W_{t}\right\|^{2}+\lambda_{2} \sum_{t=1}^{T}\left\|W_{t}-\frac{1}{T} \sum_{s=1}^{T} W_{s}\right\|_{2}^{2} \\
& \text { s.t. } e_{t}^{j}=y_{t}^{j}-\left\langle W_{t}, x_{t}^{j}\right\rangle, t=1, \cdots, T ; j=1, \cdots, \mathrm{N}_{t} .
\end{aligned}
$$

Then the augmented Lagrangian function in Eq. (12) is defined as:

$$
\begin{aligned}
\min _{W, e, \alpha, \tau} & L(e, W, \alpha, \tau)=\sum_{t=1}^{T} \sum_{j=1}^{\mathrm{N}_{t}}\left[y_{t}^{j} e_{t}^{j}\right]_{+}+\lambda_{1} \sum_{t=1}^{T}\left\|W_{t}\right\|^{2} \\
+ & \frac{\tau}{2} \sum_{t=1}^{T} \sum_{j=1}^{\mathrm{N}_{t}}\left(\left\langle W_{t}, x_{t}^{j}\right\rangle+e_{t}^{j}-y_{t}^{j}+\frac{\alpha_{t}^{j}}{\tau}\right)^{2} \\
+ & \lambda_{2} \sum_{t=1}^{T}\left\|W_{t}-\frac{1}{T} \sum_{s=1}^{T} W_{s}\right\|_{2}^{2}
\end{aligned}
$$

where $\alpha$ is the Lagrange multiplier.

At the $k$-th iteration, when parameter matrix $W$ is fixed, Eq. (13) is reduced to the following problem:

$$
\min _{e} \sum_{t=1}^{T} \sum_{j=1}^{\mathrm{N}_{t}}\left[y_{t}^{j} e_{t}^{j}\right]_{+}+\frac{\tau}{2} \sum_{t=1}^{T} \sum_{j=1}^{\mathrm{N}_{t}}\left(\left\langle W_{t}, x_{t}^{j}\right\rangle+e_{t}^{j}-y_{t}^{j}+\frac{\alpha_{t}^{j}}{\tau}\right)^{2}
$$

The optimization of Eq. (14) can be decomposed into $\sum_{t=1}^{T} \mathrm{~N}_{t}$ independent single variable minimization problem, and each is a single variable 2-piece piecewise function [33]. After the deduction, we get the explicit solution of variable $e$ :

$$
e_{t}^{j}=\left\{\begin{array}{l}
t_{t}^{j}, \text { if } y_{t}^{j} t_{t}^{j}<0 \\
0, \text { if } 0 \leq y_{t}^{j} t_{t}^{j} \leq \frac{1}{\tau} \\
t_{t}^{j}-\frac{1}{\tau} y_{t}^{j}, \text { if } y_{t}^{j} t_{t}^{j} \geq \frac{1}{\tau}
\end{array}\right.
$$

where $t_{t}^{j}=y_{t}^{j}-\left\langle X_{t}^{j}, W_{t}\right\rangle_{t}-\frac{\alpha_{t}^{j}}{\tau}, t=1, \cdots, T ; j=1, \cdots, \mathrm{N}_{t}$.

When the auxiliary variable $e$ is fixed, Eq. (13) is reduced to the following problem:

$$
\begin{aligned}
\min _{W} \lambda_{1} \sum_{t=1}^{T}\left\|W_{t}\right\|^{2}+\lambda_{2} \sum_{t=1}^{T}\left\|W_{t}-\frac{1}{T} \sum_{s=1}^{T} W_{s}\right\|_{2}^{2} \\
+\frac{\tau}{2} \sum_{t=1}^{T} \sum_{j=1}^{N_{t}}\left(\left\langle W_{t}, x_{t}^{j}\right\rangle+e_{t}^{j}-y_{t}^{j}+\frac{\alpha_{t}^{j}}{\tau}\right)^{2}
\end{aligned}
$$

This sub-problem is convex and has closed form solution. The closed form solution of parameter $W$ can be derived by letting the gradient of Eq. (16) equal to zero. 


$$
\begin{aligned}
W_{t}=- & \left(2 \lambda_{1}+2 \lambda_{2}-\frac{2 \lambda_{2}}{T}\right)^{-1}\left[\tau x_{t}\left(e_{t}-y_{t}+\frac{\alpha_{t}}{\tau}\right)\right. \\
& \left.-\frac{2 \lambda_{2}}{T} \sum_{i=1, i \neq t}^{T} W_{i}\right], t=1, \cdots, T
\end{aligned}
$$

where $x_{t}=\left[x_{t}^{1}, \cdots, \mathrm{x}_{t}^{\mathrm{N}_{t}}\right]$ is the feature matrix of $t$-th image. $e_{t}=\left[e_{t}^{1}, \cdots, e_{t}^{\mathrm{N}_{t}}\right]^{T}$ is the vector form of auxiliary variable in $t$-th image. Besides, $y_{t}=\left[y_{t}^{1}, \cdots, y_{t}^{\mathrm{N}_{t}}\right]^{T}$ is the label vector and $\alpha_{t}=\left[\alpha_{t}^{1}, \cdots, \alpha_{t}^{\mathrm{N}_{t}}\right]^{T}$ is the vector form of Lagrange multiplier.

The update of Lagrangian multiplier $\alpha$ is:

$$
\alpha_{t}^{(k)}=\alpha_{t}^{(k-1)}+\tau^{(k-1)}\left(\left\langle x_{t}, W_{t}\right\rangle+e_{t}-y_{t}\right), t=1, \cdots, \mathrm{T}
$$

The augmented penalty parameter $\tau$ is monotonically non-decreasing over the iterative process and has an upper bound. It can be updated by following formula:

$$
\tau^{(k)}=\rho \tau^{(k-1)}
$$

where $\rho \geq 1$ is a hyperparameter.

The overview of the algorithm is illustrated in Algorithm 2.

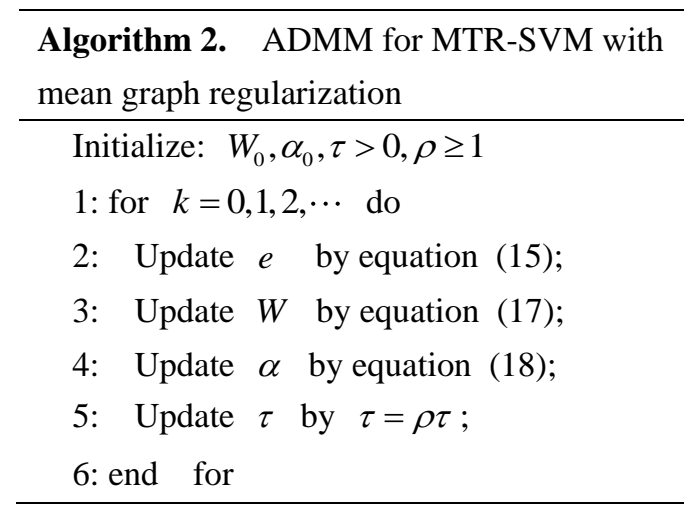

4.3 ADMM to solve problem in Eq. (8) with low rank regularization

In this part, we derive the optimization problem in Eq. (8) with the low rank regularization: $R(W)=\|W\|_{*}$.

First, we use the auxiliary variable $e_{t}^{j}=y_{t}^{j}-\left\langle W_{t}, x_{t}^{j}\right\rangle, t=1, \cdots, T ; j=1, \cdots, \mathrm{N}_{t}$ and $U=W$ to transform Eq. (8) into following form:

$$
\begin{aligned}
\min _{W} \sum_{t=1}^{T} \sum_{j=1}^{\mathrm{N}_{t}}\left[y_{t}^{j} e_{t}^{j}\right]_{+}+\lambda_{1} \sum_{t=1}^{T}\left\|W_{t}\right\|^{2}+\lambda_{2}\|U\|_{*} \\
\text { s.t. } U=W \\
e_{t}^{j}=y_{t}^{j}-\left\langle W_{t}, x_{t}^{j}\right\rangle, t=1, \cdots, T ; j=1, \cdots, \mathrm{N}_{t} .
\end{aligned}
$$

Then, the augmented Lagrangian function of Eq. (20) is defined as: 


$$
\begin{aligned}
\min _{W, e, \alpha, \tau} & L(e, W, \mathrm{U}, \alpha, \tau)=\sum_{t=1}^{T} \sum_{j=1}^{\mathrm{N}_{t}}\left[y_{t}^{j} e_{t}^{j}\right]_{+}+\lambda_{1} \sum_{t=1}^{T}\left\|W_{t}\right\|^{2} \\
+ & \frac{\tau}{2} \sum_{t=1}^{T} \sum_{j=1}^{\mathrm{N}_{t}}\left(\left\langle W_{t}, x_{t}^{j}\right\rangle+e_{t}^{j}-y_{t}^{j}+\frac{\alpha_{t}^{j}}{\tau}\right)^{2}+\lambda_{2}\|U\|_{*} \\
+ & \frac{\tau}{2}\left\|U-W+\frac{\beta}{\tau}\right\|_{F}^{2}
\end{aligned}
$$

At the $k$-th iteration, when parameter matrix W is fixed, Eq. (21) is reduced to the following two sub-problems:

$$
\begin{gathered}
\min _{e} \sum_{t=1}^{T} \sum_{j=1}^{\mathrm{N}_{t}}\left[y_{t}^{j} e_{t}^{j}\right]_{+}+\frac{\tau}{2} \sum_{t=1}^{T} \sum_{j=1}^{\mathrm{N}_{t}}\left(\left\langle W_{t}, x_{t}^{j}\right\rangle+e_{t}^{j}-y_{t}^{j}+\frac{\alpha_{t}^{j}}{\tau}\right)^{2} \\
\min _{U} \frac{\tau}{2}\left\|U-W+\frac{\beta}{\tau}\right\|_{F}^{2}+\lambda_{2}\|U\|_{*}
\end{gathered}
$$

The sub-problem in Eq. (22) is equal to Eq. (14), and it has the sane solution with Eq. (14). For the sub-problem in Eq. (23), according to [36], its globally optimal solution is given by the singular value thresholding

$$
U=Z \sum_{\frac{\lambda_{2}}{\tau}} V
$$

where $W-\frac{\beta}{\tau}=Z \sum V$ is the SVD of $W-\frac{\beta}{\tau}$, and $\sum_{\frac{\lambda_{2}}{\tau}}=\operatorname{Diag}\left\{\left(\sum_{i i}-\frac{\lambda_{2}}{\tau}\right)_{+}\right\}$.

When auxiliary $e$ and parameter matrix $U$ are fixed, Eq. (21) is reduced to the following sub-problem:

$$
\begin{aligned}
\min _{W} & \lambda_{1} \sum_{t=1}^{T}\left\|W_{t}\right\|^{2}+\frac{\tau}{2} \sum_{t=1}^{T} \sum_{j=1}^{\mathrm{N}_{t}}\left(\left\langle W_{t}, x_{t}^{j}\right\rangle+e_{t}^{j}-y_{t}^{j}+\frac{\alpha_{t}^{j}}{\tau}\right)^{2} \\
+ & \frac{\tau}{2}\left\|U-W+\frac{\beta}{\tau}\right\|_{F}^{2}
\end{aligned}
$$

The sub-problem in Eq. (25) is convex and has closed form solution. The solution of parameter matrix W can be derived by letting the gradient of Eq. (25) equal to zero,

$$
\begin{aligned}
W_{t}= & \left(2 \lambda_{1}+\tau x_{t} x_{t}^{T}+\tau\right)^{-1}\left[\tau\left(U_{t}+\frac{\beta_{t}}{\tau}\right)\right. \\
& \left.-\tau x_{t}\left(e_{t}-y_{t}+\frac{\alpha_{t}}{\tau}\right)\right], t=1, \cdots, T
\end{aligned}
$$

where $x_{t}=\left[x_{t}^{1}, \cdots, \mathrm{x}_{t}^{\mathrm{N}_{t}}\right]$ is the feature matrix of $t$-th image. $e_{t}=\left[e_{t}^{1}, \cdots, e_{t}^{\mathrm{N}_{t}}\right]^{T}$ is the vector form of auxiliary variable in $t$-th image. Besides, $y_{t}=\left[y_{t}^{1}, \cdots, y_{t}^{\mathrm{N}_{t}}\right]^{T}$ is the label vector, $\alpha_{t}=\left[\alpha_{t}^{1}, \cdots, \alpha_{t}^{\mathrm{N}_{t}}\right]^{T}$ is the vector form of Lagrange multiplier and $\beta_{t}$ is the $t$-th column of $\beta$.

The update rule of Lagrangian multiplier is: 


$$
\begin{gathered}
\alpha_{t}^{(k)}=\alpha_{t}^{(k-1)}+\tau^{(k-1)}\left(\left\langle x_{t}, W_{t}\right\rangle+e_{t}-y_{t}\right), t=1, \cdots, \mathrm{T} \\
\beta^{(k)}=\beta^{(k-1)}+\tau^{(k-1)}\left(U^{(k-1)}-W^{(k-1)}\right)
\end{gathered}
$$

The augmented penalty parameter $\tau$ is monotonically non-decreasing over the iterative process and has an upper bound. It can be updated by following formula:

$$
\tau^{(k)}=\rho \tau^{(k-1)}
$$

where $\rho \geq 1$ is a hyperparameter.

The overview of the algorithm is illustrated in Algorithm 3.

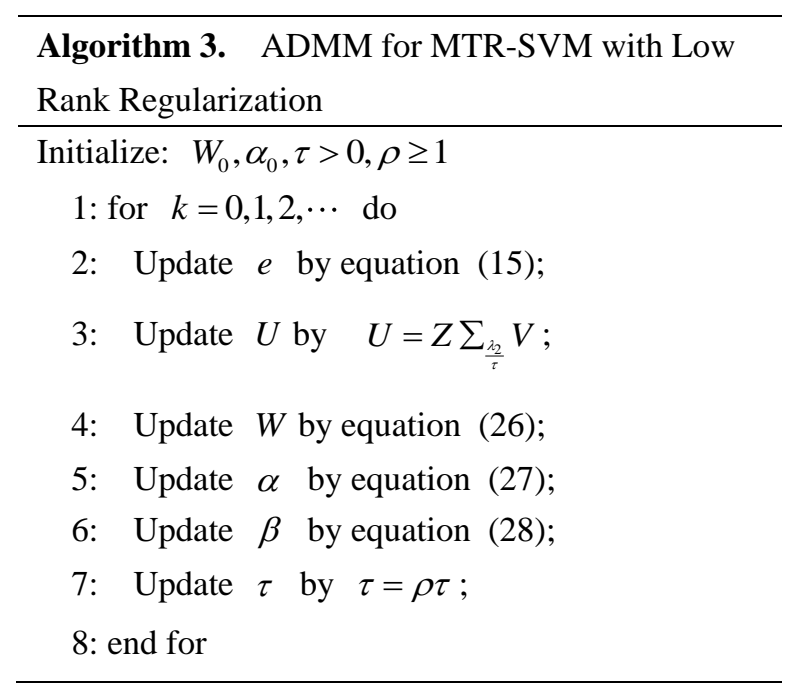

4.4 ADMM to solve problem in Eq. (8) with $\ell_{2,1}$ regularization

In this part, we derive the optimization problem in Eq. (8) with the $\ell_{2,1}$ regularization: $R(W)=\|W\|_{2,1}$. First, we use the auxiliary variable $e_{t}^{j}=y_{t}^{j}-\left\langle W_{t}, x_{t}^{j}\right\rangle, t=1, \cdots, T ; j=1, \cdots, \mathrm{N}_{t}$ and $U=W$ to transform Eq. (8) into the following form:

$$
\begin{aligned}
\min _{W} \sum_{t=1}^{T} \sum_{j=1}^{\mathrm{N}_{t}}\left[y_{t}^{j} e_{t}^{j}\right]_{+}+\lambda_{1} \sum_{t=1}^{T}\left\|W_{t}\right\|^{2}+\lambda_{2}\|U\|_{2,1} \\
\text { s.t. } e_{t}^{j}=y_{t}^{j}-\left\langle W_{t}, x_{t}^{j}\right\rangle, t=1, \cdots, T ; j=1, \cdots, \mathrm{N}_{t} . \\
\quad U=W .
\end{aligned}
$$

Then, the augmented Lagrangian function of Eq. (30) is defined as:

$$
\begin{aligned}
\min _{W, \alpha, \tau} & L(e, W, \alpha, \tau)=\sum_{t=1}^{T} \sum_{j=1}^{\mathrm{N}_{t}}\left[y_{t}^{j} e_{t}^{j}\right]_{+}+\lambda_{1} \sum_{t=1}^{T}\left\|W_{t}\right\|^{2} \\
+ & \frac{\tau}{2} \sum_{t=1}^{T} \sum_{j=1}^{\mathrm{N}_{t}}\left(\left\langle W_{t}, x_{t}^{j}\right\rangle+e_{t}^{j}-y_{t}^{j}+\frac{\alpha_{t}^{j}}{\tau}\right)^{2}+\lambda_{2}\|U\|_{2,1} \\
+ & \frac{\tau}{2}\left\|U-W+\frac{\beta}{\tau}\right\|_{F}^{2}
\end{aligned}
$$


At the $k$-th iteration, when parameter matrix W is fixed, Eq. (31) is reduced to the following two sub-problems:

$$
\begin{gathered}
\min _{e} \sum_{t=1}^{T} \sum_{j=1}^{\mathrm{N}_{t}}\left[y_{t}^{j} e_{t}^{j}\right]_{+}+\frac{\tau}{2} \sum_{t=1}^{T} \sum_{j=1}^{\mathrm{N}_{t}}\left(\left\langle W_{t}, x_{t}^{j}\right\rangle+e_{t}^{j}-y_{t}^{j}+\frac{\alpha_{t}^{j}}{\tau}\right)^{2} \\
\min _{U} \frac{\tau}{2}\left\|U-W+\frac{\beta}{\tau}\right\|_{F}^{2}+\lambda_{2}\|U\|_{2,1}
\end{gathered}
$$

The sub-problem in Eq. (32) is equal to Eq. (14) and its solution is the same with Eq. (15). For the sub-problem in Eq. (33), according to [37], its global optimal solution is given by,

$$
U_{g}=\left(1-\frac{\lambda_{2}}{\tau\left\|V_{g}\right\|}\right)_{+} V_{g}
$$

where, $V=W-\frac{\beta}{\tau}, U_{g}$ means the $g$-th row of matrix $U$ and $V_{g}$ means the $g$-th row of matrix $V$.

When the auxiliary variable $e$ and $U$ is fixed, Eq. (31) is reduced to the following sub-problem:

$$
\begin{aligned}
\min _{W} & \frac{\tau}{2} \sum_{t=1}^{T} \sum_{j=1}^{\mathrm{N}_{t}}\left(\left\langle W_{t}, x_{t}^{j}\right\rangle+e_{t}^{j}-y_{t}^{j}+\frac{\alpha_{t}^{j}}{\tau}\right)^{2}+\lambda_{1} \sum_{t=1}^{T}\left\|W_{t}\right\|^{2} \\
+ & \frac{\tau}{2}\left\|U-W+\frac{\beta}{\tau}\right\|_{F}^{2}
\end{aligned}
$$

The sub-problem in Eq. (35) is equal to Eq. (25) and its solution is the same with Eq. (26). Finally, the updating rule of Lagrangian multiplier and augmented penalty parameter are separately:

$$
\begin{gathered}
\alpha_{t}^{(k)}=\alpha_{t}^{(k-1)}+\tau^{(k-1)}\left(\left\langle x_{t}, W_{t}\right\rangle+e_{t}-y_{t}\right), t=1, \cdots, \mathrm{T} \\
\beta^{(k)}=\beta^{(k-1)}+\tau^{(k-1)}\left(U^{(k-1)}-W^{(k-1)}\right) \\
\tau^{(k)}=\rho \tau^{(k-1)}
\end{gathered}
$$

where $\rho \geq 1$ is a hyperparameter.

The overview of the algorithm is illustrated in Algorithm 4.

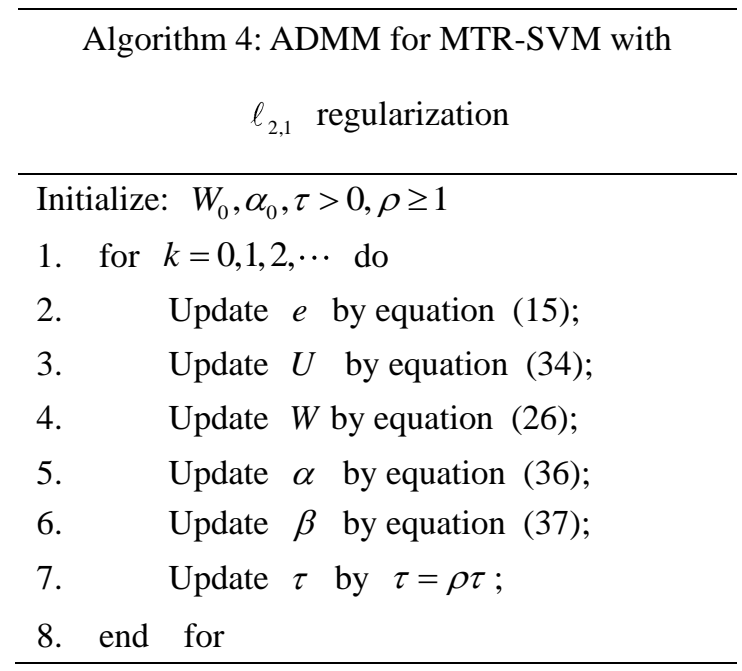




\section{Experiments}

In this section, we test the performance of the proposed MTR-SVM algorithm on two publicly available datasets, CMU iCoseg dataset and MSRC dataset. These two datasets are both wildly used by previous cosegmentation approaches to evaluate segmentation performance. The proposed method aims at improving the accuracy of cosegmentation and reducing the computation time. Thus we separately evaluate these two points on the above two datasets. The segmentation accuracy is used to quantitatively analyze the segmentation results, and it is defined as the percentage of the well classified pixels (foreground and background) over manually labeled ground truth ( $p=\frac{G T \cap R}{G T \bigcup R}$ ). In the following, three symbols, 'Mean MTL', 'Low rank MTL' and 'L2,1 MTL' are used to represent the three models used in [12].

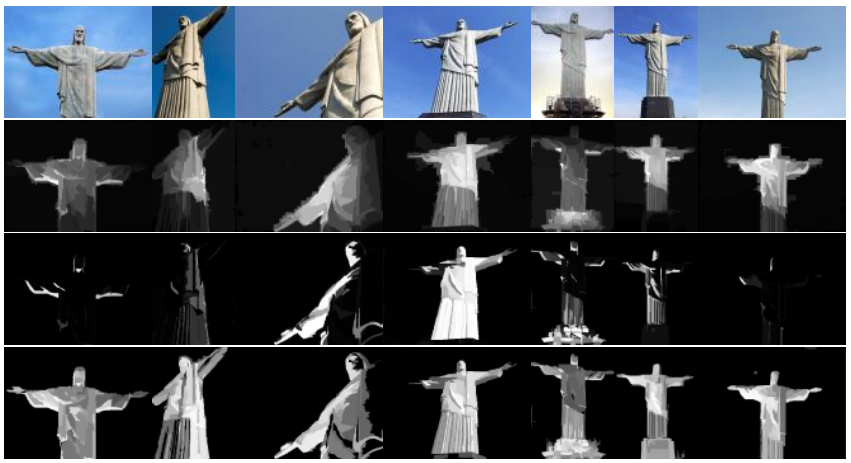

Fig. 2 The original images of Christ are presented in the first row; in the second row the saliency map are displayed, the output map of MTL are presented in the third row; in the last row the output map of the proposed approach are displayed.

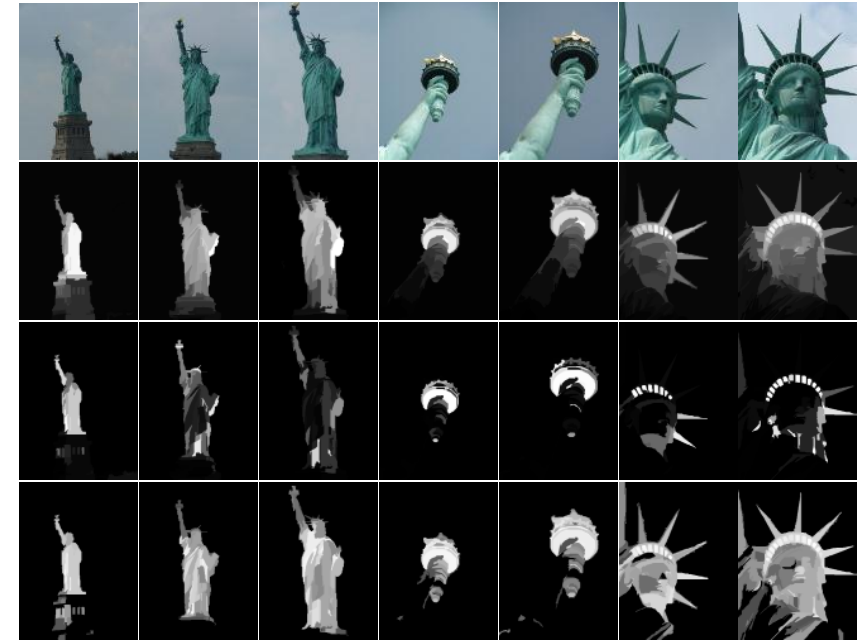

Fig. 3 The original images of Statue are presented in the first row; in the second row the saliency map are displayed, the output map of MTL are presented in the third row; in the last row the output map of the proposed approach are displayed.

\subsection{Experiments on CMU iCoseg dataset}

In this part, we evaluate the segmentation accuracy and computation time of the proposed method on CMU iCoseg dataset, which is composed of 38 object classes and contains 4 to 41 images in each image class. We fixed the parameter $\lambda_{1}$ to 0.01 for the proposed MTR-SVM with three different multi-task regularization terms. The qualitative result on iCoseg dataset is displayed in Table 1. For the comparison experiments, we rerun the codes provided by the authors on a PC with $3.6 \mathrm{GHz}$ CPU and $12 \mathrm{~GB}$ RAM and record the running time of these compared approaches. 


\begin{tabular}{|c|c|c|c|c|c|c|c|c|c|c|c|}
\hline & \multirow{2}{*}{ Class } & \multirow{2}{*}{$\begin{array}{l}\text { \# of images } \\
\text { (used/total) }\end{array}$} & \multirow{2}{*}{ [2] } & \multirow{2}{*}{ [38] } & \multirow{2}{*}{ [39] } & \multicolumn{3}{|c|}{ MTL } & \multicolumn{3}{|c|}{ MTR-SVM } \\
\hline & & & & & & Mean & LR & $\mathrm{L} 2,1$ & Mean & LR & $\mathrm{L} 2,1$ \\
\hline 1 & Alaskan bear & $19 / 19$ & 74.83 & 70.95 & 70.76 & 83.79 & 84.41 & 83.56 & 82.43 & 83.57 & 83.93 \\
\hline 2 & Red sox players & $25 / 25$ & 59.55 & 66.19 & 72.57 & 97.20 & 97.22 & 97.00 & 93.95 & 94.22 & 94.29 \\
\hline 3 & Stonehenge & $5 / 5$ & 55.66 & 70.82 & 72.19 & 78.62 & 79.01 & 78.23 & 89.52 & 87.48 & 89.79 \\
\hline 4 & Stonehenge2 & $18 / 18$ & 60.10 & 89.05 & 66.30 & 60.56 & 61.26 & 61.65 & 73.99 & 72.25 & 74.12 \\
\hline 5 & Liverpool & $33 / 33$ & 66.96 & 82.97 & 86.92 & 90.43 & 90.22 & 89.98 & 90.04 & 89.02 & 89.70 \\
\hline 6 & Ferrari & $11 / 11$ & 78.58 & 88.87 & 63.52 & 92.57 & 90.73 & 89.70 & 87.92 & 88.50 & 88.19 \\
\hline 7 & Taj Mahal & $5 / 5$ & 61.75 & 77.81 & 67.23 & 92.78 & 85.13 & 84.78 & 91.71 & 84.92 & 84.73 \\
\hline 8 & Taj Mahal2 & $5 / 5$ & 81.20 & 72.57 & 64.52 & 78.29 & 69.35 & 72.87 & 77.69 & 75.77 & 79.62 \\
\hline 9 & Pyramids & $10 / 10$ & 61.96 & 77.97 & 74.64 & 75.59 & 79.68 & 75.91 & 80.08 & 77.48 & 81.14 \\
\hline 10 & Elephants & $15 / 15$ & 54.64 & 62.27 & 74.17 & 80.17 & 81.89 & 79.86 & 84.37 & 86.24 & 86.36 \\
\hline 11 & Goose & $31 / 31$ & 97.28 & 83.96 & 69.71 & 77.96 & 78.76 & 79.92 & 89.73 & 78.86 & 80.24 \\
\hline 12 & Pandas & $25 / 25$ & 93.35 & 86.58 & 69.60 & 65.41 & 65.67 & 66.42 & 71.39 & 68.70 & 69.46 \\
\hline 13 & Airshows1 & $12 / 12$ & 69.20 & 92.87 & 87.56 & $\mathbf{9 8 . 3 8}$ & 98.27 & 95.94 & 95.03 & 94.96 & 95.19 \\
\hline 14 & Airshows2 & $39 / 39$ & 95.04 & 93.27 & 93.57 & 97.61 & 97.58 & 97.68 & 96.79 & 96.71 & 96.76 \\
\hline 15 & Airshows3 & $22 / 22$ & 56.67 & 85.27 & 80.81 & 99.60 & 99.57 & 99.51 & 99.13 & 99.09 & 99.08 \\
\hline 16 & Cheetah & $33 / 33$ & 58.92 & 90.21 & 86.00 & 73.99 & 73.33 & 72.20 & 76.20 & 76.24 & 76.41 \\
\hline 17 & Pandas2 & $21 / 21$ & 54.89 & 79.16 & 69.76 & 55.64 & 55.42 & 56.40 & 61.09 & 63.43 & 63.55 \\
\hline 18 & Kite 1 & $18 / 18$ & 98.14 & 70.77 & 86.60 & 95.87 & 95.08 & 95.28 & 96.44 & 95.96 & 96.03 \\
\hline 19 & Kite2 & $10 / 10$ & 59.84 & 84.49 & 68.17 & 77.90 & 77.01 & 77.12 & 82.72 & 83.16 & 83.20 \\
\hline 20 & Kite3 & $7 / 7$ & 61.74 & 81.86 & 69.10 & 73.24 & 71.08 & 73.00 & 79.09 & 78.36 & 78.72 \\
\hline 21 & Kite4 & $11 / 11$ & 93.92 & 77.37 & 70.67 & 97.73 & 97.76 & 97.89 & 97.21 & 97.15 & 97.24 \\
\hline 22 & Gymnastics1 & $6 / 6$ & 85.58 & 98.21 & 87.24 & 94.38 & 94.31 & 94.53 & 96.41 & 96.25 & 96.56 \\
\hline 23 & Gymnastics2 & $4 / 4$ & 71.73 & 92.00 & 81.59 & 95.38 & 95.69 & 94.93 & 95.73 & 96.20 & 96.18 \\
\hline 24 & Gymnastics 3 & $6 / 6$ & 76.36 & 96.91 & 81.82 & 92.54 & 92.58 & 92.96 & 95.21 & 95.44 & 96.29 \\
\hline 25 & Skating1 & $11 / 11$ & 66.39 & 80.47 & 72.45 & 81.19 & 79.06 & 78.39 & 82.45 & 83.78 & 83.54 \\
\hline 26 & Skating2 & $12 / 12$ & 94.72 & 56.86 & 88.60 & 97.67 & 97.50 & 96.83 & 96.32 & 96.30 & 96.91 \\
\hline 27 & Woman players1 & $36 / 36$ & 95.91 & 91.46 & 81.28 & 90.70 & 90.10 & 90.50 & 90.58 & 90.13 & 90.37 \\
\hline 28 & Woman players 2 & $16 / 16$ & 97.42 & 82.03 & 72.20 & 82.79 & 82.74 & 82.70 & 86.65 & 84.95 & 84.89 \\
\hline 29 & Monks & $17 / 17$ & 57.89 & 86.03 & 62.15 & 86.82 & 84.48 & 85.81 & 88.88 & 82.51 & 83.44 \\
\hline 30 & Hot balloons & $24 / 24$ & 72.26 & 84.29 & 79.76 & 92.14 & 90.78 & 89.69 & 90.26 & 92.05 & 93.55 \\
\hline 31 & Statue & $41 / 41$ & 72.32 & 89.17 & 86.60 & 78.53 & 80.54 & 78.04 & 87.90 & 86.40 & 88.55 \\
\hline 32 & Christ & $13 / 13$ & 68.20 & 86.91 & 73.60 & 70.20 & 70.49 & 70.16 & 86.13 & 85.76 & 85.66 \\
\hline 33 & Speedskating & $13 / 13$ & 94.77 & 59.08 & 78.15 & 89.82 & 91.85 & 89.60 & 93.28 & 93.22 & 93.24 \\
\hline 34 & Track field & $5 / 5$ & 55.22 & 67.57 & 66.87 & 81.72 & 81.76 & 81.14 & 82.67 & 83.99 & 83.47 \\
\hline 35 & Windmill & $18 / 18$ & 67.61 & 60.67 & 82.19 & 92.81 & 92.82 & 92.52 & 92.44 & 92.21 & 92.15 \\
\hline 36 & Kendo1 & $30 / 30$ & 72.31 & 67.16 & 68.81 & 93.76 & 92.86 & 95.31 & 95.15 & 90.30 & 93.63 \\
\hline 37 & Kendo2 & $11 / 11$ & 75.31 & 73.24 & 70.76 & 93.83 & 92.54 & 96.23 & 95.91 & 91.22 & 94.64 \\
\hline \multirow[t]{2}{*}{38} & Brown bear & $5 / 5$ & 66.70 & 87.76 & 84.15 & 77.71 & 78.09 & 79.22 & 88.37 & 83.02 & 85.16 \\
\hline & Average & & 73.34 & 80.13 & 75.86 & 85.14 & 84.67 & 84.57 & 87.79 & 86.73 & 87.53 \\
\hline
\end{tabular}


For each image class, all of the images are input into the cosegmentation algorithm. The segmentation accuracy of each image class is averaged over the selected images. It can be seen that the proposed approach achieves the best performance on most image classes. For each multi-task regularization term, the mean segmentation accuracy of the proposed MTR-SVM algorithm is higher than that of [12], which shows that the proposed method can reasonably utilize the saliency information of each image to achieve better cosegmentation results. In Figure 2 and figure 3, we show the saliency map, the output map of MTL in [12] and the output map of the proposed MTR-SVM on Christ image class and Statue image class respectively. And we can see that the proposed approach

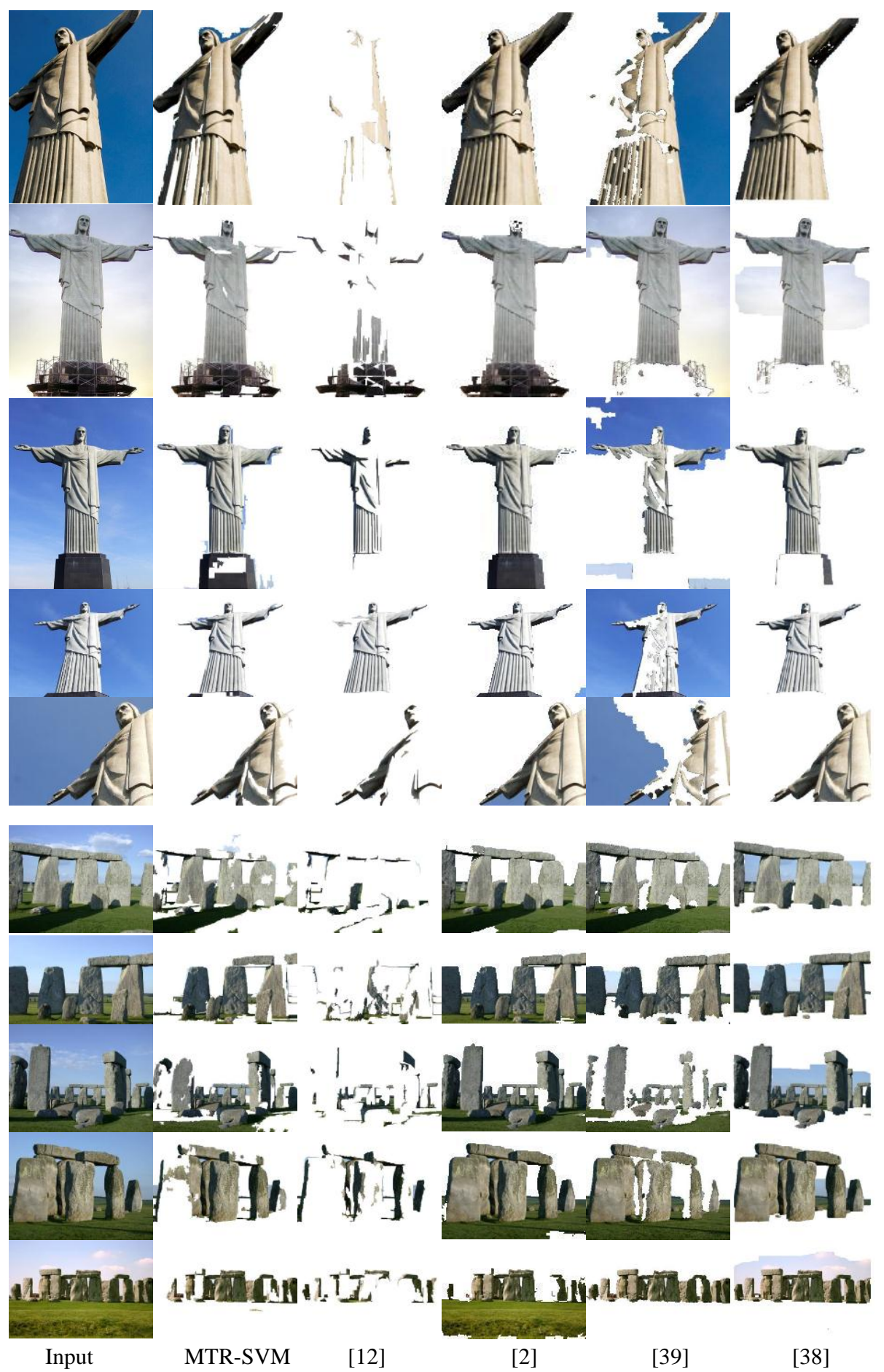

Fig. 4 The comparison of segmentation results on Christ and Stonehenge image classes. 
can achieve more bright and complete objects than the original saliency map and the output map of MTL. Thus, MTR-SVM can easily distinct the foreground objects from the background and achieve better cosegmentation results.

Figure 4 shows some segmentation examples that demonstrate the good performance of the proposed method. Figure 5 demonstrates that the proposed MTR-SVM yields fine results, even though individual images are challenging.

In the following, we evaluate the computation time of the proposed method and those compared methods. The time cost of the proposed method and those compared methods is listed in Table 2. It can be seen that [38] cost the longest time, because it used the deformable shape templates shared by input images to align common objects between these images and the computation of these shape templates is very time consuming. In [2], multiple images are modeled into one graph and a Laplace matrix must be calculated in the process of optimization. Thus, when the number of images is increasing, the time spent on the calculating of the Laplace matrix is increasing. [39] proposed a distributed cosegmentation approach for highly variable large-scale image collection, and it cost relatively little time than the above two methods. Both [12] and the proposed method are based on the framework of multi-task learning in which each image is treated as a single segmentation task. And the cosegmentation can be treated as simultaneously optimizing multiple little scale problems. Thus, compared with others methods, the multi-task learning based methods cost less time. In addition, in the proposed approach, an ADMM based optimization algorithm is proposed to optimize the MTR-SVM model and each sub-problem of ADMM has closed form solution. Thus the proposed MTR-SVM cost far less time than [12].

Table 2 Time cost on iCoseg dataset

\begin{tabular}{|c|c|c|}
\hline \multicolumn{2}{|c|}{ Method } & Time (seconds) \\
\hline \multicolumn{2}{|c|}{$[2]$} & 15300 \\
\hline \multirow{2}{|c|}[39]{} & 2123 \\
\hline \multirow{3}{*}{ MTL } & Mean & 87837 \\
\cline { 2 - 3 } & Low Rank & 666 \\
\cline { 2 - 3 } & L2,1 & 1750 \\
\hline \multirow{3}{*}{ MTR-SVM } & Low Rank & 153 \\
\cline { 2 - 3 } & Lean & 60 \\
\cline { 2 - 3 } & L2,1 & 65 \\
\hline
\end{tabular}

Table 4 Time cost on MSRC dataset

\begin{tabular}{|c|c|c|}
\hline \multicolumn{2}{|c|}{ Method } & Time (seconds) \\
\hline \multicolumn{2}{|c|}{$[2]$} & 25087 \\
\hline \multicolumn{2}{|c|}{$[3]$} & 17752 \\
\hline \multicolumn{2}{|c|}{$[39]$} & 1465 \\
\hline \multirow{3}{*}[40]{} & 93903 \\
\hline \multirow{3}{*}{ MTL } & Mean & 3029 \\
\cline { 2 - 4 } & Low Rank & 4249 \\
\cline { 2 - 4 } & L2,1 & 40 \\
\hline \multirow{3}{*}{ MTR-SVM } & Low Rank & 876 \\
\cline { 2 - 4 } & L2,1 & 17 \\
\cline { 2 - 4 } & Lean & \\
\cline { 2 - 4 } & & \\
\hline
\end{tabular}

\subsection{Experiments on MSRC dataset}

In this part, we evaluate the segmentation accuracy and computation time of the proposed method on MSRC dataset. MSRC is composed of 14 object classes and contains about 30 images in each image class. For the proposed MTR-SVM with three different multi-task regularization terms, we fixed the parameter $\lambda_{1}$ to 0.01 . The quantitative results on MSRC dataset are displayed in Table 3. For the comparison experiments, we rerun the code provided by the authors on a PC with $3.6 \mathrm{GHz}$ CPU and $12 \mathrm{~GB}$ RAM and record the running time of those compared methods.

Table 3 compares the accuracy of the proposed MTR-SVM with those conventional algorithms. For each image class, all of the images are input into the cosegmentation algorithm. The segmentation accuracy of each image class is computed by averaging the segmentation accuracy of each single image. It can be seen that the mean segmentation accuracy of [40] is the highest. Although [40] can achieve relatively high accuracy, it incorporates 
both image saliency information and the edge map information into cosegmentation. And the computation of edge map for each image cost quit a long time which is not practical in reality. For each multi-task regularization term, the mean segmentation accuracy of the proposed MTR-SVM algorithm is higher than that of [12]. In Figure 6, we show some segmentation examples on MSRC, and these examples demonstrate that the proposed MTR-SVM yields fine results, even though individual images are challenging.

Table 3 Cosegmentation performance on MSRC dataset

\begin{tabular}{|c|c|c|c|c|c|c|c|c|c|c|c|c|}
\hline & \multirow{2}{*}{ Class } & \multirow{2}{*}{$\begin{array}{l}\text { \# of images } \\
\text { (used/total) }\end{array}$} & \multirow{2}{*}{ [2] } & \multirow{2}{*}{ [3] } & \multirow{2}{*}{ [39] } & \multirow{2}{*}{ [40] } & \multicolumn{3}{|c|}{ MTL } & \multicolumn{3}{|c|}{ MTR-SVM } \\
\hline & & & & & & & Mean & LR & $\mathrm{L} 2,1$ & Mean & LR & $\mathrm{L} 2,1$ \\
\hline 1 & Bike & $30 / 30$ & 58.63 & 67.17 & 60.82 & 63.96 & 64.05 & 64.98 & 64.57 & 64.50 & 67.89 & 64.91 \\
\hline 2 & Bird & $32 / 32$ & 60.73 & 68.21 & 84.68 & 90.77 & 87.19 & 88.31 & 89.02 & 86.16 & 87.18 & 87.18 \\
\hline 3 & Car & $30 / 30$ & 75.82 & 76.59 & 65.72 & 71.50 & 68.62 & 67.79 & 68.80 & 73.13 & 73.28 & 73.79 \\
\hline 4 & Cat & $24 / 24$ & 59.29 & 66.15 & 75.64 & 82.87 & 72.89 & 73.88 & 73.91 & 78.49 & 76.75 & 73.56 \\
\hline 5 & Chair & $30 / 30$ & 76.57 & 72.54 & 70.74 & 80.96 & 72.78 & 74.89 & 75.98 & 73.61 & 73.99 & 73.39 \\
\hline 6 & Cow & $30 / 30$ & 79.03 & 83.75 & 74.74 & 91.80 & 87.89 & 88.53 & 89.76 & 87.93 & 90.22 & 88.33 \\
\hline 7 & Dog & $30 / 30$ & 76.55 & 78.20 & 70.25 & 85.58 & 79.30 & 79.96 & 81.16 & 79.50 & 76.69 & 79.29 \\
\hline 8 & Face & $30 / 30$ & 63.69 & 60.47 & 66.25 & 74.22 & 76.04 & 76.82 & 77.16 & 76.50 & 78.22 & 78.94 \\
\hline 9 & Flower & $32 / 32$ & 70.13 & 69.61 & 72.02 & 84.21 & 75.85 & 75.39 & 76.06 & 79.87 & 78.68 & 78.07 \\
\hline 10 & House & $30 / 30$ & 68.15 & 68.45 & 68.45 & 80.46 & 72.85 & 73.15 & 73.83 & 72.88 & 67.90 & 75.60 \\
\hline 11 & Plane & $30 / 30$ & 55.57 & 55.27 & 73.47 & 80.66 & 72.72 & 73.45 & 70.87 & 74.51 & 76.04 & 74.57 \\
\hline 12 & Sheep & $30 / 30$ & 80.24 & 89.24 & 74.45 & 91.81 & 87.89 & 87.64 & 88.54 & 89.87 & 89.54 & 89.86 \\
\hline 13 & Sign & $30 / 30$ & 77.83 & 76.79 & 74.22 & 84.36 & 86.76 & 85.85 & 85.82 & 83.06 & 84.39 & 84.10 \\
\hline \multirow[t]{2}{*}{14} & Tree & $30 / 30$ & 84.52 & 83.98 & 73.34 & 84.78 & 72.66 & 72.49 & 73.03 & 76.94 & 77.28 & 77.48 \\
\hline & Average & & 70.48 & 72.60 & 71.77 & 82.00 & 76.96 & 77.36 & 77.75 & 78.35 & 78.43 & 78.51 \\
\hline
\end{tabular}

The time cost of the proposed method and those compared methods is listed in Table 4, which demonstrates the efficiency of the proposed MTR-SVM approach.

\section{Conclusions and future works}

In this paper, we propose a multi-task ranking SVM model to simultaneously segment multiple images. MTR-SVM combines the framework of multi-task learning with learning to rank. It can simultaneously deal with more than two images and add different types of cosegmentation prior by the multi-task regularization term. In addition, we propose an efficient ADMM based algorithm to optimize the proposed model. Experiment results demonstrate that the proposed approach outperforms the state-of-the-art techniques significantly both on the iCoseg dataset and MSRC dataset. In future works, we look forward to exploring more multi-task regularization terms, such as nonconvex regularization and other low rank regularization [41, 42]. Furthermore, we will investigate an automatic mechanism to construct the multi-task regularization term.

\section{Acknowledgments}

This work was supported by the grants of the National Science Foundation of China, Nos. 61133010, 61520106006, 31571364, 61532008, 61572364, 61373105, 61303111, 61411140249, 61402334, 61472282, 61472280, 61472173, 61572447, and 61373098, China Postdoctoral Science Foundation Grant, Nos. 
2014M561513, and partly supported by the National High-Tech R\&D Program (863) (2014AA021502 \& 2015AA020101), and the grant from the Ph.D. Programs Foundation of Ministry of Education of China (No. 20120072110040).

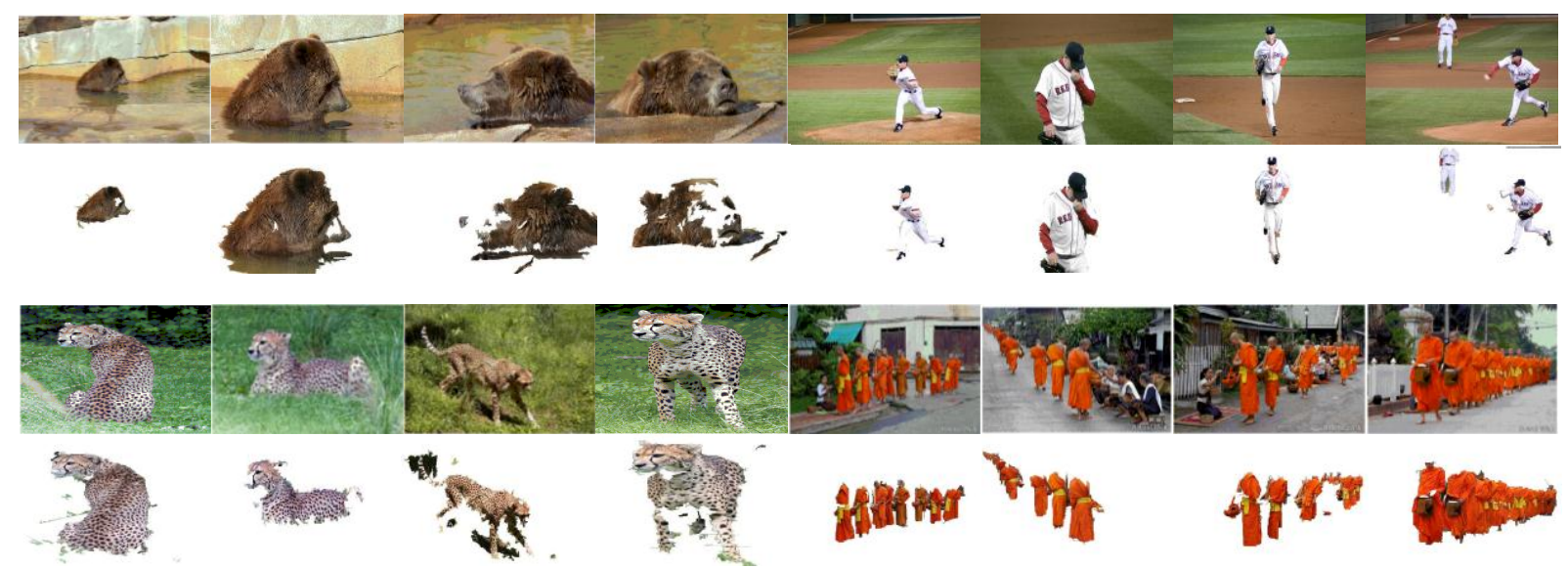

Fig. 5 Images and segmentation results on iCoseg dataset. There are 4 groups of images. In each group, the top row represents the original images, and the bottom row represents the segmentation result.

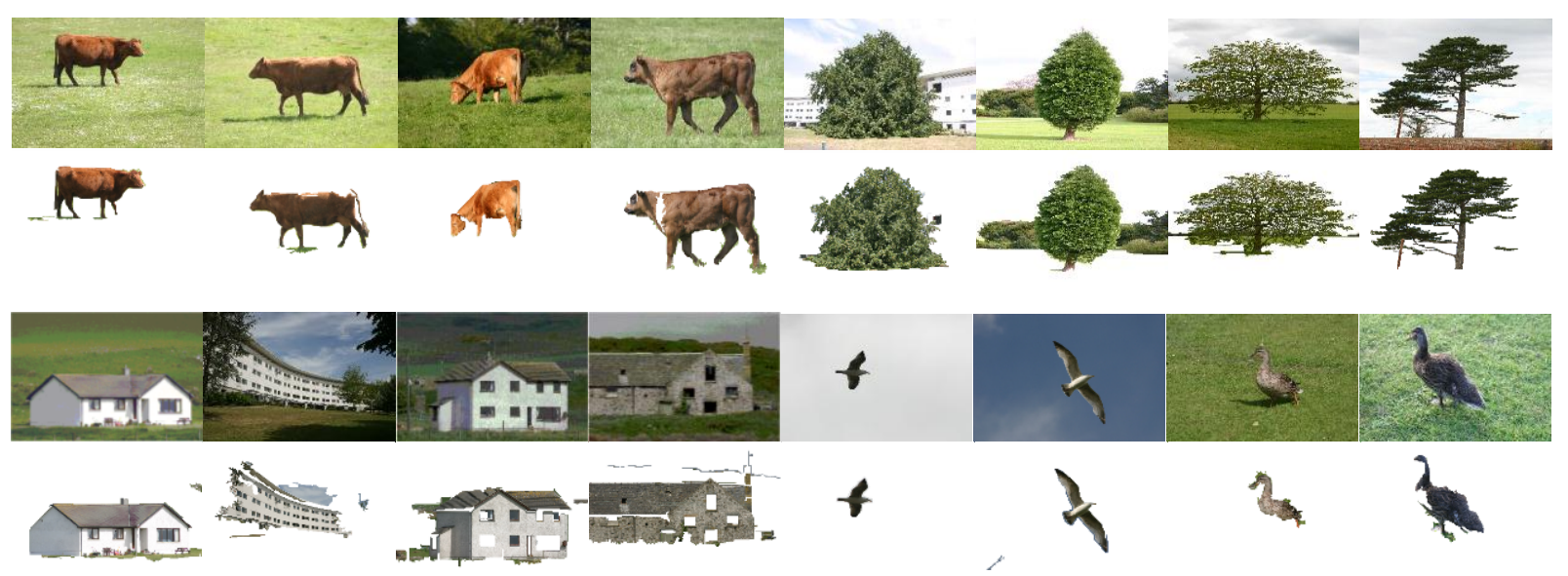

Fig. 6 Images and segmentation results on MSRC dataset. There are 4 groups of images. In each group, the top row represents the original images, and the bottom row represents the segmentation result.

\section{References}

[1] C. Rother, T. Minka, A. Blake, V. Kolmogorov, Cosegmentation of image pairs by histogram matching-incorporating a global constraint into mrfs, in: Proceedings of 2006 IEEE Conference on Computer Vision and Pattern Recognition (CVPR), IEEE, 2006, pp. 993-1000.

[2] A. Joulin, F. Bach, J. Ponce, Discriminative clustering for image co-segmentation, in: Proceedings of 2010 IEEE Conference on Computer Vision and Pattern Recognition (CVPR), IEEE, 2010, pp. 1943-1950.

[3] A. Joulin, F. Bach, J. Ponce, Multi-class cosegmentation, in: Proceedings of 2012 IEEE Conference on Computer Vision and Pattern Recognition (CVPR), IEEE, 2012, pp. 542-549.

[4] D. Kuettel, M. Guillaumin, V. Ferrari, Segmentation propagation in imagenet, in: Proceedings of 12th 
European Conference on Computer Vision (ECCV), Springer, 2012, pp. 459-473.

[5] M. Rubinstein, C. Liu, W.T. Freeman, Annotation propagation in large image databases via dense image correspondence, in: Proceedings of 12th European Conference on Computer Vision (ECCV), Springer, 2012, pp. 85-99.

[6] Y. Li, J. Liu, Z. Li, H. Lu, S. Ma, Object co-segmentation via salient and common regions discovery, Neurocomputing, 172 (2015) 225-234.

[7] R. Quan, J. Han, D. Zhang, F. Nie, Object co-segmentation via graph optimized-flexible manifold ranking, in: Proceedings of 2016 IEEE Conference on Computer Vision and Pattern Recognition (CVPR), IEEE, 2016, pp. 687-695.

[8] S. Vicente, C. Rother, V. Kolmogorov, Object cosegmentation, in: Proceedings of 2011 IEEE Conference on Computer Vision and Pattern Recognition (CVPR), IEEE, 2011, pp. 2217-2224.

[9] H. Fu, D. Xu, S. Lin, J. Liu, Object-based RGBD Image Co-segmentation with Mutex Constraint, in: Proceedings of 2015 IEEE Conference on Computer Vision and Pattern Recognition (CVPR), IEEE, 2015, pp. 4428-4436.

[10] Z. Liu, J. Zhu, J. Bu, C. Chen, Object cosegmentation by nonrigid mapping, Neurocomputing, 135 (2014) 107-116.

[11] K. Li, J. Zhang, W. Tao, Unsupervised Co-Segmentation for Indefinite Number of Common Foreground Objects, IEEE Transactions on Image Processing, 25 (2016) 1898-1909.

[12] Q. Zhang, J. Zhou, Y. Wang, J. Ye, B. Li, Image Cosegmentation via Multi-task Learning, in: Proceedings of 25th British Machine Vision Conference (BMVC), BMVA, 2014.

[13] J. Liu, S. Ji, J. Ye, Multi-task feature learning via efficient 1 2, 1-norm minimization, in: Proceedings of the 25th Conference on Uncertainty in Artificial Intelligence (UAI), AUAI, 2009, pp. 339-348.

[14] T. Evgeniou, M. Pontil, Regularized multi--task learning, in: Proceedings of the tenth ACM SIGKDD International Conference on Knowledge Discovery and Data Mining, ACM, 2004, pp. 109-117.

[15] R.K. Ando, T. Zhang, A framework for learning predictive structures from multiple tasks and unlabeled data, The Journal of Machine Learning Research, 6 (2005) 1817-1853.

[16] Y. Cao, J. Xu, T.-Y. Liu, H. Li, Y. Huang, H.-W. Hon, Adapting ranking SVM to document retrieval, in: Proceedings of the 29th Annual International ACM SIGIR Conference on Research and development in Information Retrieval, ACM, 2006, pp. 186-193.

[17] T.-Y. Liu, Learning to rank for information retrieval, Foundations and Trends in Information Retrieval, 3 (2009) 225-331.

[18] L. Hang, A short introduction to learning to rank, IEICE TRANSACTIONS on Information and Systems, 94 (2011) 1854-1862.

[19] D. Cossock, T. Zhang, Subset ranking using regression, in: Proceedings of 19th International Conference on Learning Theory, Springer, 2006, pp. 605-619.

[20] Z. Cao, T. Qin, T.-Y. Liu, M.-F. Tsai, H. Li, Learning to rank: from pairwise approach to listwise approach, in: Proceedings of the 24th international conference on Machine learning (ICML), ACM, 2007, pp. 129-136.

[21] S. Boyd, C. Cortes, M. Mohri, A. Radovanovic, Accuracy at the top, in: Proceedings of 26th Advances in Neural Information Processing Systems (NIPS), 2012, pp. 953-961.

[22] N. Li, R. Jin, Z.-H. Zhou, Top rank optimization in linear time, in: Proceedings of 28th Advances in Neural Information Processing Systems (NIPS), 2014, pp. 1502-1510.

[23] P. Jawanpuria, M. Lapin, M. Hein, B. Schiele, Efficient Output Kernel Learning for Multiple Tasks, in: Proceedings of 29th Advances in Neural Information Processing Systems (NIPS), 2015, pp. 1189-1197. 
[24] R. Achanta, A. Shaji, K. Smith, A. Lucchi, P. Fua, S. Susstrunk, SLIC superpixels compared to state-of-the-art superpixel methods, IEEE Transactions on Pattern Analysis and Machine Intelligence, 34 (2012) 2274-2282.

[25] H. Jiang, J. Wang, Z. Yuan, Y. Wu, Salient Object Detection: A Discriminative Regional Feature Integration Approach, in: Proceedings of 2013 IEEE Conference on Computer Vision and Pattern Recognition (CVPR), IEEE, 2013, pp. 2083-2090.

[26] R. Margolin, A. Tal, L. Zelnik-Manor, What makes a patch distinct?, in: Proceedings of 2013 IEEE Conference on Computer Vision and Pattern Recognition (CVPR), IEEE, 2013, pp. 1139-1146.

[27] C. Lang, G. Liu, J. Yu, S. Yan, Saliency detection by multitask sparsity pursuit, IEEE Transactions on Image Processing, 21 (2012) 1327-1338.

[28] J. Qi, S. Dong, F. Huang, H. Lu, Saliency detection via joint modeling global shape and local consistency, Neurocomputing, 222 (2016) 81-90.

[29] C. Jia, J. Qi, X. Li, H. Lu, Saliency detection via a unified generative and discriminative model, Neurocomputing, 173 (2016) 406-417.

[30] C. Rother, V. Kolmogorov, A. Blake, Grabcut: Interactive foreground extraction using iterated graph cuts, ACM transactions on graphics, 23 (2004) 309-314.

[31] D. Gabay, B. Mercier, A dual algorithm for the solution of nonlinear variational problems via finite element approximation, Computers \& Mathematics with Applications, 2 (1976) 17-40.

[32] H. Wang, F. Nie, H. Huang, Low-rank tensor completion with spatio-temporal consistency, in: Proceedings of 28th Association for the Advancement of Artificial Intelligence (AAAI), 2014, pp. 2846-2852.

[33] F. Nie, Y. Huang, X. Wang, H. Huang, New primal svm solver with linear computational cost for big data classifications, in: Proceedings of 31th International Conference on Machine Learning (ICML), IMLS, 2014, pp.505-513.

[34] C. Lu, H. Li, Z. Lin, S. Yan, Fast Proximal Linearized Alternating Direction Method of Multiplier with Parallel Splitting, in: Proceedings of 30th Association for the Advancement of Artificial Intelligence (AAAI), 2016, pp. 739-745.

[35] C. Lu, J. Feng, S. Yan, Z. Lin, A Unified Alternating Direction Method of Multipliers by Majorization Minimization, arXiv preprint arXiv:1607.02584, 2016.

[36] J.-F. Cai, E.J. Candès, Z. Shen, A singular value thresholding algorithm for matrix completion, SIAM Journal on Optimization, 20 (2010) 1956-1982.

[37] N. Parikh, S.P. Boyd, Proximal Algorithms, Foundations and Trends in optimization, 1 (2014) 127-239.

[38] J. Dai, Y.N. Wu, J. Zhou, S.-C. Zhu, Cosegmentation and cosketch by unsupervised learning, in: Proceedings of 2013 IEEE International Conference on Computer Vision (ICCV), IEEE, 2013, pp. 1305-1312.

[39] G. Kim, E.P. Xing, L. Fei-Fei, T. Kanade, Distributed cosegmentation via submodular optimization on anisotropic diffusion, in: Proceedings of 2011 IEEE International Conference on Computer Vision (ICCV), IEEE, 2011, pp. 169-176.

[40] F. Meng, H. Li, G. Liu, K.N. Ngan, Object co-segmentation based on shortest path algorithm and saliency model, IEEE transactions on multimedia, 14 (2012) 1429-1441.

[41] C. Lu, C. Zhu, C. Xu, S. Yan, Z. Lin, Generalized singular value thresholding, arXiv preprint arXiv:1412.2231, 2014.

[42] C. Lu, J. Tang, S. Yan, Z. Lin, Generalized nonconvex nonsmooth low-rank minimization, in: Proceedings of 2014 IEEE Conference on Computer Vision and Pattern Recognition (CVPR), IEEE, 2014, pp. 4130-4137. 